\title{
PROPOSTA DE MENSURAÇÃO DE RISCO BASEADO EM UTILIDADE
}

\section{RISK MEASUREMENT PROPOSAL BASED ON UTILITY}

\author{
RODRIGO LEONE \\ Professor Doutor \\ do Mestrado Profissional em Administração \\ do Departamento de Administração \\ da Universidade Potiguar - RN \\ E-mail: r.leone@uol.com.br \\ ROBERTO OUIRINO DO NASCIMENTO \\ Professor DSC \\ do Departamento de Estatística \\ do Centro de Ciências Exatas e da Natureza - CCEN \\ da Universidade Federal da Paraíba - PB \\ E-mail: quirino@de.ufpb.br
}

\author{
GEORGE GUERRA LEONE \\ Professor Doutor \\ do Departamento de Contabilidade \\ da Universidade Federal da Paraíba - PB \\ E-mail: r.leone@hotmail.com \\ PAULO OLIVEIRA \\ Professor Doutor \\ dos Programas de Pós-graduação de Engenharia - COPPE \\ da Universidade Federal do Rio de Janeiro - RJ \\ E-mail: poliveir@cos.uffj.br
}

\section{RESUMO}

A mensuração do risco, integrada aos modelos de finanças corporativas mediante o trabalho de Markowitz, era baseada na variância dos retornos em torno do retorno médio naquele período. Entretanto, apesar do cálculo do risco pela variância ter a propriedade de penalizar variações superiores a uma unidade, tal metodologia não diferencia variações negativas de variações positivas. Neste trabalho, propõe-se uma mensuração do risco, na qual se incorpora uma função utilidade, de modo a retratar a característica, suposta comum aos investidores racionais, de que a decepção com a perda é mais sentida que a satisfação com o ganho. A inserção dessa nova componente, além de fornecer novas interpretações de volatilidade, mostrou-se um instrumento de alerta mais eficaz que a metodologia usual.

Palavras-chave: Mensuração do Risco; Funçã்o Utilidade; Desvio-Utilidade; Mercado de Ações.

\section{ABSTRACT}

The measurement of risk presented in Markowitz' work was based on the variance of returns. In spite of penalizing deviation greater than the unity, this methodology does not differentiate positive from negative deviation. This study proposes a measurement of risk in which a utility function is incorporated to describe the characteristics of a rational investor. The disappointment of the investor with the loss is greater than the satisfaction with the gain. This new component, in addition to supplying other interpretations for volatility, seems to be a more effective alert instrument than the usual methodology.

Keywords: Measurement of Risk, Utility Function, Utility Deviation, Stock Market. 


\section{INTRODUÇÃO}

As empresas têm em geral o mesmo quadro de problemas, necessidades e expectativas (TELÓ, 2001). Já que operam em um ambiente de volatilidade, influenciado por fatores como: a globalização dos mercados, o aumento da flutuação das taxas de câmbio, as mudanças tecnológicas, dentre outros. Segundo Lemgruber e Ohanian (1997), fatores como o crescimento da volatilidade e liquidez dos mercados fazem com que as empresas, principalmente as do setor financeiro, invistam no desenvolvimento de instrumentos de mensuração do risco de mercado.

Por isso, verifica-se o esforço contínuo de executivos e pesquisadores no sentido de entender e gerenciar essas volatilidades para diminuir os riscos (PESSOA, 2006), já que, direta ou indiretamente, esses riscos influenciam o custo de capital dos projetos, impactando nos retornos esperados e finalmente na capacidade de criar valor para a empresa.

Nesse sentido, as questões financeiras mais relevantes dizem respeito ao orçamento de capital, à estrutura de capital e à administração do capital de giro (ROSS et al., 2002; DAMODARAN, 2002), sendo que dessas três, as decisões de investimento é o tema principal. Para auxiliar o administrador financeiro nessa tarefa, existem vários métodos de avaliação de investimentos: métodos tradicionais que não levam em conta os riscos, métodos que analisam os riscos e métodos que gerenciam os riscos.

A mensuração do risco não é tarefa simples. Tanto que a variável risco somente foi integrada aos modelos de finanças corporativas mediante o trabalho de Markowitz de 1959. No trabalho em questão, o risco para um período em estudo foi calculado com base na dispersão, medida pela variância, dos retornos em torno do retorno médio naquele período (SOUZA, 2003).

O cálculo do risco pela variância tem a propriedade de penalizar variações superiores a uma unidade, pois considera a norma euclidiana, porém não diferencia variações negativas de variações positivas. Assim sendo, uma variação de $3 \%$ além do esperado tem peso nove vezes maior do que uma variação positiva de $1 \%$. O mesmo continuaria válido, caso as variações fossem negativas. Mais ainda, o peso da variação positiva de $3 \%$ é idêntico ao da variação negativa de $3 \%$. Já para o desvio-médio, os pesos são proporcionais aos valores absolutos das variações. Mattos (1998, p.1) afirma que

[...] se a hipótese de que a distribuição de retornos dos ativos não é normal, ou se a função de utilidade do investidor não é quadrática, a variância não é medida adequada de risco, pois estabelece pesos iguais para os diversos retornos do ativo, não distinguindo se este é maior ou menor que a média.

Os modelos clássicos de otimização de carteira de investimentos a partir de um conjunto de ativos, geralmente, consideram o risco definido pelo desvio-padrão e, portanto, sofrem a mesma conseqüência apontada acima.

A informação para a tomada de decisão sobre investimento em ativos só está minimamente completa quando são dados o retorno esperado, o risco associado a esse retorno e o intervalo de confiança para esse risco. Como é de praxe utilizar-se uma probabilidade (confiança) de 95\% para o intervalo [retorno - risco; retorno + risco], inferido por uma tendência normal de comportamento para a variável retorno, basta informar o par risco/retorno. O desconhecimento desse fato pode acarretar em perdas muito além das expectativas do investidor, mas não necessariamente improváveis estatisticamente. Com os pares risco/retorno para vários ativos em mãos, quase nunca é possível ordená-los do melhor ao pior. A decisão depende do perfil do investidor: o mais agressivo opta por ativos de maior volatilidade, cujos retornos são geralmente mais altos, correndo o risco de ganhos ainda maiores ou de perdas catastróficas, enquanto o mais conservador se satisfaz com retornos menores, de preferência com baixo risco de perda. É a lei da compensação.

No decorrer do trabalho, será considerada válida a seguinte hipótese central sobre um investidor conservador: a decepção com a perda é mais sentida que a satisfação com o ganho. Exemplificando, um retorno de $-1 \%$ quando se esperava $2 \%$ não é compensado por um retorno de $5 \%$ para a mesma expectativa. Oscilações negativas são mais influentes que oscilações positivas e, portanto, devem assumir maior ponderação (LAUGHHUNN e CRUM, 1980; MAO, 1970).

Neste artigo, o objetivo é propor um cálculo para a mensuração do risco (em distribuições simétricas), levando em conta a característica (hipótese central) suposta comum aos investidores racionais (conservadores) comentada anteriormente. Para tanto, introduzir-se-á uma função utilidade, dependente dos parâmetros média e desvio-padrão, de modo a desconsiderar observações discrepantes e penalizar perdas com peso superior ao peso de privilegiar ganhos. Considera-se, ainda, a existência de um parâmetro positivo de modo a abranger vários perfis de investidores.

O trabalho está dividido da seguinte forma: na fundamentação teórica, realiza-se um levantamento dos trabaIhos mais recentes envolvendo a Mensuração do Risco e com breves comentários sobre Modelagem e Gerenciamento de Risco e Função Utilidade. Em seguida, introduz-se a noção de Distância-Utilidade, essencial para a boa definição de Desvio-Utilidade. Posteriormente, apresentam-se e avaliam-se os resultados experimentais para a metodologia de mensuração de risco proposta. Finalmente, serão apresentadas algumas considerações sobre a utilização da metodologia proposta. 


\section{MODELAGEM E GERENCIAMENTO DO RISCO}

Ao longo do século $X X$, surgiram vários métodos de auxílio à tomada de decisão sobre investimentos (ROSS et al., 2002; YOUNG e O.BYRNE, 2003; BREALEY e MYERS, 2000; BRIGHAM et al. 2001; TRIGEORGIS, 1996). Alguns deles supõem um cenário sem riscos e outros, um cenário com risco. Dentre os que levam em conta o fator risco, há aqueles que apenas o mensuram, aqueles que o analisam $e$, finalmente, aqueles que o administram.

Atualmente, o tema central da maioria dos trabalhos envolvendo risco diz respeito a seu gerenciamento, tanto em empresas financeiras, quanto em não-financeiras (DUARTE, 1996; JORION, 2000). Sobre essas últimas, podem-se citar (BANDEIRA-DE-MELLO e CUNHA, 2004), em que os autores apresentam e utilizam a teoria substantiva para entender a administração estratégica do risco em ambientes turbulentos; (FAMÁ et al., 2001), que tomam como base a metodologia do Balanced Scorecard (KAPLAN e NORTON, 1997) para propor um modelo de gestão integrada do risco; já Kimura e Perera (2003) elaboram um modelo de otimização da gestão de riscos, identificando uma estratégia de hedge que maximiza a esperança dos lucros e Saito e Schiozer (2004 e 2005) que apresentam uma pesquisa evidenciando o uso de derivativos pelos executivos brasileiros principalmente com o propósito de gerenciar risco e não com fins especulativos.

No tocante ao risco de mercado, destaca-se o trabalho de Almeida e Ghirardi (1999), que compara três modelos de estimativa da volatilidade para o cálculo do VaR (Value at Risk) e o de Machado e Pinto (2004), que apresenta uma metodologia para tratar as situações de estresse no mercado financeiro, caracterizadas por ocorrências extremas na distribuição dos retornos. Finalmente, o trabalho de La Rocque e Lowenkron (2004), advertindo que a gestão eficaz dos riscos de mercado depende do tipo de entidade interessada e que sua prática ainda é incipiente, principalmente devido a sua difícil adaptabilidade à realidade das corporações.

A implementação do gerenciamento de risco deve ser uma decisão de quem efetivamente detém o poder decisório na instituição. Devem-se buscar profissionais qualificados e experientes para essa tarefa, pois um mau gerenciamento de risco pode levar a uma falsa sensação de segurança, o que pode ser até mesmo pior que desconhecer - e, portanto, temer - o risco de suas posições hoje. A confiabilidade da estimativa final obtida para o risco de uma instituição está diretamente relacionada à qualidade dos dados usados e dos procedimentos computacionais implementados (DUARTE, 1996).

Damodaran (2002, p.56) indica que um bom modelo de risco e retorno

a. deve proporcionar uma medida de risco que se aplique a todos os ativos, não sendo específica apenas a um deles;

b. deve estabelecer claramente quais tipos de risco são recompensados e quais não são, assim como fornecer um fundamento lógico para essa distinção;

c. deve proporcionar medidas de risco padronizadas, isto é, um investidor diante de uma medida de risco para um ativo específico deve ser capaz de tirar conclusões a respeito de se o ativo está acima ou abaixo da média em termos de risco;

d. deve traduzir a medida de risco em uma "taxa de retorno" que o investidor deve exigir como compensação por assumir o risco;

e. deve funcionar bem não apenas ao explicar retornos passados, mas também ao prever retornos futuros.

Os modelos mais comuns (KOSKOSIDIS e DUARTE, 1997), com menor ou maior grau de complexidade, por exemplo, o Modelo de Média-Variância de Markowitz, o Modelo de Precificação de Ativos de Capital - CAPM (TREYNOR apud ROSS, 2002; SHARPE, 1964; LINTNER, 1965) e a Teoria de Precificação por Arbitragem (ROSS, 1976), satisfazem a todos esses requisitos, com a ressalva de apenas recompensarem o risco de mercado (DAMODARAN, 2002).

A próxima seção contém uma breve apresentação da Teoria de Utilidade, que servirá de base para a construção do modelo proposto neste trabalho.

\section{A TEORIA DE UTILIDADE}

A Teoria de Utilidade encontra-se amplamente difundida e é utilizada em diversas áreas do conhecimento, entre elas, Economia (MONTORO et al., 1998; HEILBRONER, 1978), Telecomunicações (SARAYDAR et al., 2000; SARAYDAR et al., 2001) e Finanças (DUARTE, 1997). Seu conceito baseia-se no princípio da saturabilidade (SANDRONI, 1994) e, por questões didáticas, é comum apresentá-lo ligado apenas à propensão ou satisfação em consumir (MONTORO et al., 1998; HEILBRONER, 1978), mas pode-se, perfeitamente, estendê-lo ao desprazer de não consumir.
Örerler (2006) apresenta três tipos de atitudes envolvendo risco, modeladas por funções utilidade. Seu estudo empírico é baseado em teorias comportamentais. Mattos (1998, p.1) afirma que

[...] a noção de risco do investidor assemelha-se mais ao chamado risco de perda, ou seja, o risco de perder uma determinada quantia, do que ao risco de variabilidade do retorno do ativo, como expresso pela variância.

Para sustentar a afirmação, cita os estudos de Kahneman e Tversky (1979) e de Hogarth (1988), que defendem 
o uso de medidas de risco focadas na possibilidade de perda e não na dispersão total, tendem a retratar mais fielmente o comportamento humano. Cita, também, o estudo de Laughhunn e Crum (1980) sobre a atitude ante o risco para retornos abaixo de uma meta preestabelecida; e o de Mao (1970), em que diversos gerentes americanos, quando perguntados sobre o que entendiam pelo termo "risco do investimento" responderam que risco se refere primariamente aos desvios abaixo da taxa de retorno alvo e que nunca se preocuparam com o retorno do projeto quando está acima do retorno alvo.

Leone (2004) dá um exemplo corriqueiro de ganho e perda marginal, identifica entre seus agentes, o investidor, o retorno esperado no período e a possibilidade de retornos maiores ou menores que o esperado, traduzida (a possibilidade) pelo desvio-padrão. Assim, conclui que variações positivas em relação ao retorno esperado seriam bem menos sentidas que oscilações negativas, cada vez em maior grau.

\section{PROPOSTA DE RISCO BASEADO EM UTILIDADE}

Nessa seção, será apresentada uma proposta de cálculo de risco baseado em uma função utilidade parametrizada pela média dos retornos e pelo desvio-padrão do índice de mercado. Assim como no CAPM, considerar-se-á uma distribuição de freqüências simétrica (normal) para o comportamento dos retornos.

Como explicado anteriormente, o objetivo é inserir na mensuração do risco um fator capaz de traduzir o fato de que a decepção com a perda é mais sentida que a satisfação com o ganho, ou seja, que oscilações negativas são mais influentes que oscilações positivas e, portanto, devem assumir maior ponderação (LAUGHHUNN e CRUM, 1980; MAO, 1970).

Definição 1: $O$ desvio-padrão amostral (ou populacional) de referência é um desvio-padrão representativo do mercado em que está inserida a amostra.

Por exemplo, para ativos negociados na BOVESPA, o desvio-padrão de referência é o desvio-padrão das oscilações do lbovespa.

Definição 2: Uma amostra $A$ é dita Amostra Referencial Válida se:

i. $\bar{x}+6 \sigma>0 \mathrm{e}$

ii. $P(Y) \geq 90 \%$, em que $Y=\left\{X_{i} \in A ; X_{i}+6 \sigma>0\right.$, para $i=1, \ldots, n\}$,

em que: $n$ é o número de observações na amostra; $X_{i}$ é a observação; $\bar{X}$ é a média; $\sigma$ é o desvio-padrão amostral de referência e $P(Y)$ é a probabilidade associada ao conjunto $Y$.

Definição 3: $A$ Função-Utilidade definida em $A$ é dada por

$$
U(X)=\rho \ln \left(\frac{X+6 \sigma}{\bar{X}+6 \sigma}\right)
$$

em que: $X$ é uma observação e $\bar{X}$ é a média da amostra referencial válida; $\sigma$ é o desvio-padrão amostral (ou populacio- nal) de referência e $\rho$ é um parâmetro positivo que depende do perfil do investidor, apenas quanto ao risco.

Sobre essa última definição é interessante evidenciar as seguintes considerações:

- tomam-se, apenas, amostras referenciais válidas, pois ativos com $\bar{X}+6 \sigma \leq 0$ têm diminutas possibilidades de retorno positivo;

- ainda sobre amostra referencial válida, mesmo satisfazendo $\bar{X}+6 \sigma>0$, só serão considerados os ativos tais que $X+6 \sigma>0$, para $90 \%$ das observações (as observações complementares na amostra serão descartadas). Os ativos que não satisfazem essa premissa também têm pouca chance de originar retornos positivos.

Diante das considerações indicadas anteriormente, observa-se que:

- o quociente entre parênteses é sempre positivo e o logaritmo está bem definido;

- as propriedades dessa função se justapõem ao comportamento desejado para a avaliação das observações. Dentre outras, destaca-se que o valor absoluto do logaritmo para variações negativas cresce mais rápido que o valor absoluto do logaritmo para variações positivas em relação à média;

- em (DUARTE, 1997), o autor propõe uma função utilidade linear dada pela soma de uma wealth function linear (função riqueza) com uma função penalidade, também linear, em que essa última é aplicada apenas para retornos (ou riquezas) abaixo do mínimo aceitável. Com essa metodologia, o autor dá maior peso às variações negativas do que às variações positivas, porém mantendo-o constante.

Definição 4: $A$ Distância-Utilidade para $x, y \in A$ é o número real dado por:

$$
d(x, y)=\left\{\begin{array}{lll}
d(x, \bar{X})-d(y, \bar{X}), & \text { para } x \leq y \leq \bar{X} & \text { ou } \bar{X} \leq y \leq x \\
d(y, \bar{X})-d(x, \bar{X}), & \text { para } y \leq x \leq \bar{X} & \text { ou } \bar{X} \leq x \leq y \\
d(x, \bar{X})+d(y, \bar{X}), & \text { para } x \leq \bar{X} \leq y & \text { ou } y \leq \bar{X} \leq x
\end{array}\right.
$$


em que:

$$
\begin{aligned}
d(x, \bar{X}) & =(x-\bar{X})^{2}[U(x)]^{2} \\
& =(x-\bar{X})^{2} \operatorname{In}^{2}\left(\frac{X+6 \sigma}{\bar{X}+6 \sigma}\right)
\end{aligned}
$$

$\forall x \in A$ e $\rho=1$.

As seguintes observações são relevantes:

- o número real $d(x, \bar{X})$ é positivo para todo $x \in A$, $\operatorname{com} x \neq \bar{x}$

- Considera-se $\rho=1$, para facilitar o entendimento, porém as definições e proposição dessa seção continuam válidas para qualquer $\rho>0$.

Proposição 1: A Distância-Utilidade satisfaz às propriedades de distância (LIMA, 1993):
d1) $d(x, x)=0$
d2) Se $x \neq y$, então $d(x, y)>0$;
d3) $d(x, y)=d(y, x) e$
d4) $d(x, z) \leq d(x, y)+d(y, z)$.

Demonstração: ver (LEONE, 2004, p.34-38)

Definição 5: A Variância-Utilidade $\left(V A R_{U}\right)$ é o número real dado por:

$$
V A R_{U}=\frac{1}{n} \cdot \sum_{i=1}^{n} d\left(X_{i}, \bar{X}\right),
$$

em que: $n$ é a quantidade de observações para as quais a função-utilidade está bem definida, isto é, as observações em que o logaritmo existe.

Definição 6: O Desvio-Utilidade (DU) é a raiz quadrada da Variância-Utilidade.
Observações:

- a unidade do Desvio-Utilidade é a mesma unidade original, podendo, portanto, ser comparada com a média da amostra;

- pela proposição d2, $D U \geq 0$, para qualquer conjunto de dados;

- mesmo se tratando de amostras, considera-se $n$ e não $n$-1. A teoria necessária para explicar essa possível diferenciação no denominador não faz parte do objetivo deste trabalho.

Para melhor entendimento, considere-se o exemplo da Tabela 10 para retornos de dois ativos com média e desvio-padrão já calculados.

Calculando, para cada um deles, o desvio-utilidade, com base em um desvio-padrão de referência arbitrário de $3 \%$, têm-se os resultados apresentados na Tabela $2 \mathbf{0}$.

Do quadro anterior, percebe-se que o afastamento entre as observações no Ativo $A$ e sua média são sempre maiores que o afastamento entre as observações do Ativo $B$ e sua média. Tal fato acarreta em um maior valor de desvio-padrão para o Ativo A: 6,60\% contra 5,40\%. Apesar disso, o desvio-utilidade do Ativo $B$ é bem maior do que o do Ativo $A$ : $11,31 \%$ contra $4,42 \%$. Mais ainda, o valor obtido para mensurar o risco do Ativo $A$ quando se leva em conta a utilidade foi inferior ao valor obtido pela maneira usual. O inverso aconteceu para o Ativo B: o valor obtido para mensurar seu risco quando há influência da utilidade foi superior ao valor obtido quando tal fato não é levado em conta. O exemplo evidencia, portanto, a interferência do fator logarítmico no cálculo de risco via desvio-utilidade, fazendo com que, como desejado, as oscilações negativas tenham maior peso do que as oscilações positivas.

Tabela 1

\begin{tabular}{c|c|c}
\hline${\text { Observação } \mathbf{n}^{\mathbf{0}}}$ & Retornos Ativo A & Retornos Ativo B \\
\hline 1 & $-2,0 \%$ & $2,0 \%$ \\
\hline 2 & $-2,0 \%$ & $2,0 \%$ \\
\hline 3 & $-2,0 \%$ & $2,0 \%$ \\
\hline 4 & $-2,0 \%$ & $2,0 \%$ \\
\hline 5 & $-2,0 \%$ & $2,0 \%$ \\
\hline 6 & $-2,0 \%$ & $2,0 \%$ \\
\hline 7 & $-2,0 \%$ & $2,0 \%$ \\
\hline 8 & $-2,0 \%$ & $2,0 \%$ \\
\hline 9 & $-2,0 \%$ & $2,0 \%$ \\
\hline 10 & $20 \%$ & $-16 \%$ \\
\hline Retorno Médio $\left(\mathbb{R}_{\mathrm{M}}\right)$ & $0,20 \%$ & $0,20 \%$ \\
\hline Desvio-Padrão $|\sigma|$ & $6,60 \%$ & $5,40 \%$
\end{tabular}

Tabela 2

\begin{tabular}{c|c|c} 
Ativo & Desvio-Padrão & Desvio-Utilidade \\
\hline A & $6,60 \%$ & $4,42 \%$ \\
\hline B & $5,40 \%$ & $11,31 \%$
\end{tabular}


Propriedade 1: Os valores $X_{p}$, tais que:

$$
\frac{\bar{X}+6 \sigma(e-1)}{e}<X_{i}<\bar{X} e+6 \sigma(e-1)
$$

contribuem para um desvio-utilidade menor que o desviopadrão, em que $\bar{X}$ é a média da amostra referencial válida; $\sigma$ é o desvio-padrão de referência e e é o neperiano.

Demonstração:

$$
\begin{aligned}
& \frac{\bar{X}+6 \sigma(e-1)}{e}<X_{i}<\bar{X} e+6 \sigma(e-1) \\
\Leftrightarrow & \bar{X}-6 \sigma e+6 \sigma<X_{i} e<\bar{X} e^{2}+6 \sigma e(e-1) \\
\Leftrightarrow & \bar{X}+6 \sigma<\left(X_{i}+6 \sigma\right) e<(\bar{X}+6 \sigma) e^{2} \\
\Leftrightarrow & \frac{1}{e}<\frac{X_{i}+6 \sigma}{\bar{X}+6 \sigma}<e \\
\Leftrightarrow & \operatorname{In}\left(\frac{1}{e}\right)<\operatorname{In}\left(\frac{X_{i}+6 \sigma}{\bar{X}+6 \sigma}\right)<\operatorname{In}(e) \\
\Leftrightarrow & -1<\operatorname{In}\left(\frac{X_{i}+6 \sigma}{\bar{X}+6 \sigma}\right)<1 \\
\Leftrightarrow & 0<\operatorname{In}^{2}\left(\frac{X_{i}+6 \sigma}{\bar{X}+6 \sigma}\right)<1
\end{aligned}
$$

Daí, para todo $X_{i}$ satisfazendo à condição enunciada, resultará:

$$
\begin{aligned}
& \left(X_{i}-\bar{X}\right)^{2} \operatorname{In}^{2}\left(\frac{X_{i}+6 \sigma}{\bar{X}+6 \sigma}\right) \leq\left(X_{i}-\bar{X}\right)^{2} \\
\Rightarrow & \frac{1}{n} \sum_{i=1}^{n}\left(X_{i}-\bar{X}\right)^{2} I^{2}\left(\frac{X_{i}+6 \sigma}{\bar{X}+6 \sigma}\right) \leq \frac{1}{n} \sum_{i=1}^{n}\left(X_{i}-\bar{X}\right)^{2}
\end{aligned}
$$

Tirando a raiz quadrada, tem-se que o desvio-padrão é cota superior para o desvio-utilidade.
Propriedade 2: Para $\bar{X}>3 \sigma\left(\frac{2-e}{e-1}\right), \bar{x} e+3 \sigma(e-1)$ é sempre discrepante pelo critério do Escore Z. Para $\bar{X}>$ $\frac{3 \sigma}{e-1}, \frac{\bar{x} e-3 \sigma(e-1)}{e}$ é discrepante.

Demonstração: Basta comparar $\bar{X} e+3 \sigma(e-1)$ com $\bar{x}+3 \sigma e \frac{\bar{x} e-3 \sigma(e-1)}{e} \operatorname{com} \bar{x}-3 \sigma$.

\subsection{Comentários ao Modelo Proposto}

A medida de risco obtida via desvio-utilidade, claramente, apenas leva em conta o risco de mercado, porém se aplica a qualquer ativo, desde que se consiga um desviopadrão de referência, satisfazendo, portanto, aos requisitos (a.) e (b.) da seção 1. Como a unidade de desvio-utilidade é a unidade original dos retornos, pode-se entender a informação resultante como uma "taxa de retorno" cobrada para se assumir risco, com o adicional de se considerarem as utilidades marginais de perdas e ganhos. Como a distribuição de freqüências das oscilações para a metodologia proposta é induzida pela distribuição de freqüências para a metodologia usual, supor que essa última tem tendência normal permite fazer inferências e avaliar as probabilidades para futuras oscilações via desvio-utilidade. Assim, os requisitos (d.) e (e.) podem ser considerados como satisfeitos. Para serem completados os requisitos de um bom modelo, satisfazendo o item (c.), define-se o risco médio como o desvio-utilidade do mercado no qual estão inseridos os ativos em estudo. No caso de ativos negociados na BOVESPA, as medidas de risco estariam padronizadas pelo desvio-utilidade do índice Ibovespa para o período em questão.

\section{RESULTADOS EXPERIMENTAIS}

Para avaliar o desvio-utilidade como medida de risco, procede-se a dois testes: no primeiro, simplesmente calcula-se o desvio-utilidade para três "ativos" com diferentes níveis de risco medido pelo desvio-padrão, no intuito de averiguar se o resultado confirmaria ou não a classificação obtida por essa última metodologia. O segundo teste preocupou-se em avaliar o desvio-utilidade como instrumento de alerta contra períodos de quedas acentuadas nos retornos mensais de alguns ativos negociados na BOVESPA.

\subsection{Desvio-Utilidade com Medida de Volatilidade}

Para ser atingido o objetivo proposto, coletam-se os retornos mensais dos "ativos" Poupança, IBOVESPA, Petrobras PN de janeiro de 1996 a março de 2004. A poupan- ça foi escolhida por, segundo Silveira et al. (2002, p.10), "comportar-se de acordo com a definição teórica da taxa livre de risco no que diz respeito à sua correlação com outros ativos existentes na economia", já que depende unicamente da Taxa Referencial de Juros (TR) e do número de dias úteis no mês. Seu desvio-padrão no período em estudo foi de $0,30 \%$ para um retorno médio mensal de 0,98\%. O índice IBOVESPA pode ser considerado um "ativo" arriscado, porém, por se tratar de uma carteira diversificada, conta apenas com o chamado risco sistemático. Seu desvio-padrão para o período foi de $10,67 \%$ contra $2,27 \%$ ao mês de retorno médio. Já o ativo Petrobras PN foi escolhido por contar com os chamados risco sistemático e risco nãosistemático, já que sua rentabilidade está sujeita tanto aos fatores do mercado como um todo, quanto aos fatores ine- 
rentes a seu setor, tais como o preço do barril de petróleo e a cotação do dólar. Seu desvio-padrão no período foi de $13,60 \%$ contra $3,77 \%$ ao mês de retorno médio.

A Tabela $3 \mathbf{O}$ apresenta os resultados para o desvioutilidade.

A explicação para o alto valor do risco para o ativo Petrobras PN está nos retornos mensais do período entre agosto de 1997 a dezembro de 1998. Nesse período, apesar de o índice IBOVESPA também ter sofrido duras quedas em alguns meses, as quedas no valor da ação Petrobras PN foram, em geral, mais acentuadas: $-34,29 \%$ contra $-23,83 \%$ em outubro de 1997 e $-52,94 \%$ contra $-39,55 \%$ em agosto 1998.

Deve-se observar que a avaliação do desvio-utilidade não deve ser efetuada comparando-se esse resultado com o desvio-padrão isoladamente. $O$ fato de ter-se desvio-padrão igual a 10,67\% e desvio-utilidade igual a 4,99\% para o IBOVESPA, assim como $13,60 \%$ e $11,51 \%$, não significa que 0 instrumento desvio-utilidade avaliou esses ativos como menos arriscados que via desvio-padrão, afinal de contas, são instrumentos medidos, por definição, em escalas diferentes. A avaliação deve pressupor o conjunto de resultados, comparando não só o desvio-padrão com o desvio-utilidade para cada ativo, como também o quociente entre os desvios-padrão dos ativos e o quociente entre os desvios-utilidade. Além, obviamente, de comparar esses desvios com o retorno médio.

\subsection{Desvio-Utilidade como Instrumento de Alerta}

Para esse segundo teste, coletam-se os retornos de 20 empresas listadas na BOVESPA, assim como a pontuação média mensal e a de fechamento do mês do índice IBO-
VESPA e a rentabilidade mensal da poupança, de janeiro de 1996 a março de 2004.

O Gráfico 10 apresenta o comportamento da pontuação média mensal do índice IBOVESPA no período estudado.

Com o auxílio do Gráfico 1, podem-se identificar os períodos de maiores quedas na pontuação do índice. A avaliação do desvio-utilidade, como instrumento de alerta para períodos de queda acentuada nas cotações dos ativos coletados, baseou-se nesses períodos críticos, que se detalham a seguir:

a. julho/1997 - novembro/1997: Desvalorizações em diversos "tigres asiáticos" (Tailândia, Filipinas, Malásia, Coréia). No dia 23 de outubro, acontece uma forte queda na Bolsa de Valores de Honk Kong, provocando, durante a semana seguinte, quedas acentuadas em todas as Bolsas no mundo. No Brasil, o governo eleva a taxa de juros básica (SELIC) de 21\% para 43\%, para garantir taxa câmbio;

b. abril/1998 - janeiro/1999: Dia 7/08, Crise da Rússia (desvalorização e moratória) em agosto de 1998. Forte queda da Bolsa de Nova York. No Brasil, onde o governo mantinha o câmbio valorizado, apesar das fortes pressões, acontece o "setembro negro". Nesse período, após fortes saídas de recursos (em alguns dias cerca de US\$ 3 bilhões), o governo decide elevar a Taxa SELIC de $19 \%$ para $28,75 \%$ e, em seguida, para $49,75 \%$. Para acentuar a crise, há especulações sobre a possibilidade de impeachment do Presidente Clinton e uma piora das expectativas sobre a capacidade de o governo defender a moeda;

c. agosto/2000 - novembro/2000: No final do terceiro trimestre, novas indefinições em relação ao cenário internacional, decorrentes da crise do petróleo e da

Tabela 3

\begin{tabular}{c|c|c|c} 
& Poupança & IBOVESPA & Petrobras PN \\
\hline Desvio-Utilidade & $0,0029 \%$ & $4,99 \%$ & $11,51 \%$
\end{tabular}

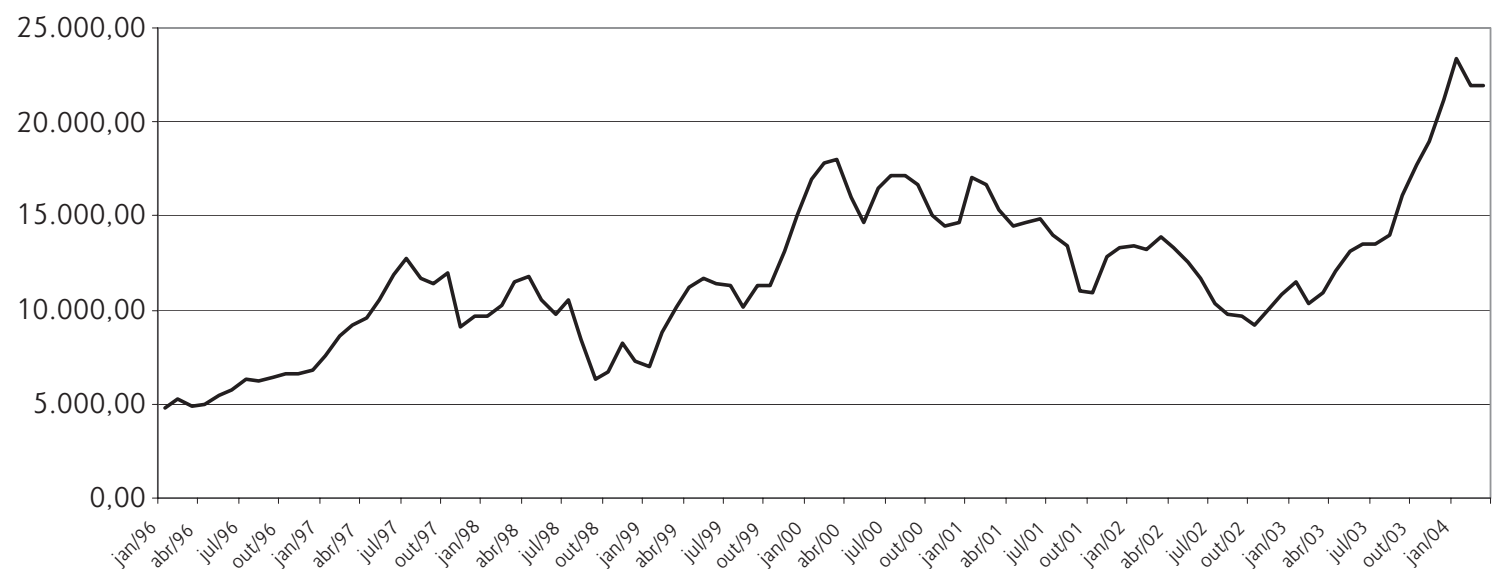

Gráfico 1 
evolução dos fundamentos macroeconômicos na Argentina e nos EUA. Tais fatores são percebidos na curva da estrutura a termo das taxas de juros, indicando o temor dos investidores em relação à possibilidade de recessão nos EUA;

d. janeiro/2001 - outubro/2001: Ciclo recessivo da economia mundial, o fim do processo de esvaziamento da bolha especulativa da Nova Economia, os atentados terroristas de 11 de setembro nos Estados Unidos e a maior falência da história de uma empresa dos EUA e o racionamento de energia iniciado em junho contribuem para a performance sofrível do mercado em 2001: o valor de mercado das companhias listadas na BOVESPA atingiu US $\$ 142,8$ bilhões em setembro, queda de $36,7 \%$ no ano, resultado da combinação entre a queda no preço das ações e a depreciação cambial;

e. março/2002 - outubro/2002: Turbulência no mercado e desconfiança do investidor estrangeiro, já abalado pela fraude contábil da WorldCom na Bolsa americana. Perspectiva de ansiedade quanto às novas pesquisas de intenção de votos e quanto à possibilidade de contágio da economia brasileira pela crise Argentina, impactando no câmbio, na taxa de juros e nos indicadores de inflação.

Para esses cinco períodos relacionados, o procedimento de investigação foi o seguinte: calcular o desvio-padrão e o desvio-utilidade, para os 20 ativos coletados, nos seis meses anteriores ao mês subseqüente à primeira queda acentuada no índice IBOVESPA; calcular a perda acumulada nos meses seguintes à primeira queda acentuada do índice IBOVESPA. De posse dessas informações, classificam-se os ativos em ordem decrescente de desvio-padrão e de desvio-utilidade. Os resultados estão para os cinco ativos mais arriscados segundo cada uma das metodologias resumidas na Tabela $4 \mathbf{0}$.

Pode-se verificar que há pouca diferença entre os resultados obtidos. Apesar disso, tem-se alguma sustentação para concluir que, para os ativos selecionados e para os períodos estudados, o desvio-utilidade mostrou-se uma ferramenta de alerta mais eficaz contra a escolha de ativos de rentabilidade potencialmente negativa.

Com base nos mesmos resultados, tentou-se classificar o ativo como agressivo, moderado ou conservador, tanto para a metodologia de risco pelo desvio-padrão quanto para a metodologia pelo desvio-utilidade.

Um ativo foi considerado agressivo no período em estudo pela metodologia de desvio-padrão se possuía desvio-padrão no período superior ao desvio-padrão do IBOVESPA no mesmo período; foi considerado conservador se possuía desvio-padrão no período inferior ao dobro do desvio-padrão da poupança no mesmo período; finalmente, foi considerado moderado se não fosse nem agressivo, nem conservador. $O$ mesmo procedimento foi utilizado para classificar os ativos como agressivo, moderado ou conservador, segundo a metodologia de risco via desvioutilidade, com a ressalva de serem comparados os respectivos desvios-utilidade com o desvio-utilidade do IBOVESPA e com o dobro do desvio-utilidade da poupança, sempre no período referente. A Tabela $5 \boldsymbol{O}$ apresenta os resultados (número de ativos considerados agressivos entre os 20 ativos selecionados) para as duas metodologias, para cada um dos períodos estudados.

Para todos os períodos estudados, a metodologia de mensuração de risco pelo desvio-utilidade considerou um número maior de ativos arriscados dentre os 20 ativos selecionados. Tal fato indica que o acréscimo do fator logarítmico na fórmula do desvio-utilidade torna, como se pressupunha, a proposta de cálculo de risco mais conser-

Tabela 4

\begin{tabular}{c|c|c} 
Período & $\begin{array}{c}\text { Resultado Acumulado } \\
\text { Metodologia Desvio-Padrão }\end{array}$ & $\begin{array}{c}\text { Resultado Acumulado } \\
\text { Metodologia Desvio-Utilidade }\end{array}$ \\
\hline jul./1997 - nov./1997 & $-90,03 \%$ & $-93,91 \%$ \\
\hline abr./1998 - jan./1999 & $67,09 \%$ & $-49,49 \%$ \\
\hline ago./2000 - nov./2000 & $-31,68 \%$ & $-28,76 \%$ \\
\hline jan./2001 - out./2001 & $-98,83 \%$ & $-107,59 \%$ \\
\hline mar./2002 - out./2002 & $-69,88 \%$ & $-69,88 \%$
\end{tabular}

Tabela 5

\begin{tabular}{l|c|c} 
& $\begin{array}{c}\text { \# Ativos Agressivo } \\
\text { (Desvio-Padrão) }\end{array}$ & $\begin{array}{c}\text { \# Ativos Agressivo } \\
\text { (Desvio-Utilidade) }\end{array}$ \\
\hline abr./1997 a set./1997 & 6 & 9 \\
\hline abr./1998 a set./1998 & 7 & 11 \\
\hline abr./2000 a set./2000 & 17 & 18 \\
\hline nov./2000 a abr./2001 & 10 & 14 \\
\hline nov./2001 a abr./2002 & 8 & 11
\end{tabular}


vadora que a metodologia de risco pelo desvio-padrão. Ao classificar um número maior de ativos como agressi- vos, o desvio-utilidade inibe o investimento nesses ativos arriscados.

\section{CONSIDERAC̣ÕES FINAIS}

O artigo propôs uma nova metodologia de avaliação de risco, que combina norma euclidiana com uma função utilidade logarítmica. Essa função tem a propriedade de atribuir maior peso às oscilações negativas do que às oscilações positivas. Assim, tem-se um indicador mais sensível para o risco de um ativo.

Aplicando a proposta de desvio-utilidade a um conjunto de ativos negociados na BOVESPA, foi obtida uma classificação quanto ao risco diferente da classificação usual, calculada com base no desvio-padrão. Essas classificações selecionaram ativos cujo somatório das perdas acumula- das foi, na maioria das vezes, menor se originado da classificação via desvio-utilidade. Além disso, os resultados obtidos indicam que a metodologia de desvio-utilidade é mais conservadora que a metodologia de desvio-padrão, pois definiu, para todos os casos estudados, um percentual maior de ativos como agressivos.

Como não se tem teoria suficiente que justifique a adoção do desvio-utilidade, não se pode abandonar a(s) metodologia(s) usual(is). Porém, acredita-se que a proposta deva se estabelecer como análise complementar às analises existentes.

\section{Referências Bibliográficas}

ALMEIDA, A. F. de; GHIRARDI, A.. Estudo Comparativo de Modelos de Gerenciamento de Risco de Mercado com uma Carteira Composta por Ativos Típicos de um Fundo de Ações. In: ENANPAD, 23, 1999, Foz do Iguaçu/PR. Anais... Foz do Iguaçu/PR, ANPAD, 1999, 1, CD-ROM.

BANDEIRA-DE-MELLO, R.; CUNHA, C. J. C. de A.. Administrando o Risco: uma Teoria Substantiva da Adaptação Estratégica de Pequenas Empresas a Ambientes Turbulentos e com Forte Influência Governamental. RAC, Edição Especial, v.8, p.157-179, 2004.

BREALEY, R. A; MYERS, S. C.. Principles of Corporate Finance. $6^{\text {th }}$ ed.. Mc Graw-Hill, 2000.

BRIGHAM, E. F; GAPENSKI, L. C.; EHRHARDT, M. C.. Administração Financeira - Teoria e Prática. São Paulo: Atlas, 2001.

DAMODARAN, A.. Finanças Corporativas Aplicadas: Manual do Usuário. Porto Alegre: Bookman, 2002.

DUARTE Jr., A. M.. Risco: Definições, Tipos, Medição e Recomendações para seu Gerenciamento. Resenha BMßF, v.114, p.25-33, 1996.

A framework for the active management of a global currency fund. Revista de Econometria, v.17, n.2, p.1-19, 1997.

FAMÁ, R.; CARDOSO, R. L.; MENDONÇA NETO, O.. Gestão Integrada de Riscos para Empresas Não Financeiras: Uma Proposta de Modelo para Controladoria, In: ENANPAD, 25, 2001, Campinas/SP. Anais... Campinas/SP, ANPAD, 2001, 1,CD-ROM.

HEILBRONER, R. L.. Elementos de Macroeconomia. 4a ed.. Rio de Janeiro: Zahar Editores, 1978.

HOGARTH, R.. Judgement and Choice. John Wiley \& Sons Ltd., Essex, 1988.

JORION, P.. Value at Risk - The New Benchmark for Managing Financial Risk. 2º ed. New York: MacGraw-Hill, 2000.

KAHNEMAN, D.; TVERSKY, A.. Prospect Theory: An Analysis of Decisions under Risk. Econometrica, 1979.

KAPLAN, R. S.; NORTON, D. P.. A Estratégia em Ação. Rio de Janeiro: Campus, 1997.

KIMURA, H.; PERERA, L. C. J.. Modelamento Ótimo da Gestão de Risco em Empresas Não financeiras, In: ENANPAD, 27, 2003, Atibaia/SP. Anais... Atibaia/SP, ANPAD, 2003, 1, CD ROM.

KOSKOSIDIS, Y. A.; DUARTE Jr., A. M.. A Scenario-Based Approach to Active Asset Allocation. Journal of Portfolio Management, v.23, p.74-85, 1997.

LA ROCQUE, E.; LOWENKRON, A.. Métricas e Particularidades da Gestão de Risco em Corporações. Risk Control, 2004.

LAUGHHUNN, D. P.; CRUM, R.. Managerial Risk Preferences for Below-Target Returns. Management Science, v.26, n.12, p.1238-1249, 1980.

LEMGRUBER, E. F.; OHANIAN, G.. O Modelo de Projeção de Volatilidade do RiskMetrics ${ }^{\mathrm{TM}}$ e a Hipótese de Distribuição Normal Condicional Para Alguns Fatores de Risco do Brasil. In: ENANPAD, 21, 1997, Rio das Pedras/RJ. Anais... Rio das Pedras/RJ, ANPAD, 1997, 1, CD-ROM.

LEONE, R. J. G.. Modelagem e Otimização de um Sistema de Telecomunicação sem fio e de uma Carteira de Investimentos. 2004. Tese. (Doutorado em Engenharia de Sistemas e Computação) - COPPE/UFRJ, Rio de Janeiro, RJ.

LIMA, E. L.. Espaços Métricos. Rio de Janeiro: Livros Técnicos e Científicos, 1993.

LINTNER, J.. The valuation of risk assets and the selection of risky investments in stock portfolios and capital budgets. Review of Economics and Statistics, 1965.

MACHADO, S. J.; PINTO A. C. F.. Controlando o Risco de Mercado de um Fundo de Previdência Privada: Um Modelo Integrado de VaR e Teste de Estresse Sob a Ótica da Teoria do Valor Extremo In: ENANPAD, 28, 2004, Curitiba. Anais... Curitiba/PR, ANPAD, 2004, 1, CD-ROM.

MAO, J.. Models of Capital Budgeting. E-V Versus E-S. Journal of Financial and Quantitative Analysis, v.4, p.657-675, 1970. 
MATTOS, A. E. K.. O uso do Downside Risk (Média - Momentos Parciais) como medida de risco na seleção de portfolios. In: ENANPAD, 22, 1998, Foz do Iguaçu/PR. Anais... Foz do Iguaçu/PR, ANPAD, 1998, 1, CD-ROM.

MONTORO, A. F. FILHO et al.. In: PINHO, D. B.; VASCONCELOS, M. A. S. (Organizadores). Manual de Economia, $3^{\text {a }}$ ed.. São Paulo: Saraiva, 1998.

PESSOA, G. A.., Avaliação de projetos de mineração utilizando a teoria das opções reais em tempo discreto: um estudo de caso em mineração de ferro. 2006. Dissertação (Mestrado em Administração) - FGV/EBAPE, Rio de Janeiro, RJ.

ÖRERLER E. O.. Utility Function and Risk Taking: an experiment. The Journal of the American Academy of Business, v.9, n.2, p.167-174, 2006. ROSS, S. A.. The arbitrage theory of capital asset pricing. Journal of Economic Theory, 1976.

ROSS, S. A.; WESTERFIELD, R. W; JAFFE, J. F.. Administração Financeira - Corporate Finance, São Paulo: Atlas, 2002.

SANDRONI, P.. Novo Dicionário de Economia, São Paulo: Best Seller, 1994.

SARAYDAR, C. U.; MANDAYAN, N. B.; GOODMAN, D. J.. Efficient power control via pricing in wireless data networks, IEEE Trans. Comm., aceito, 2000.

SARAYDAR, C. U.; MANDAYAN, N. B.; GOODMAN, D. J.. Pricing and Power Control Modeling in a Multicell Wireless data network. IEEE Journal on Select Areas in Communications, Special Issue: mobility and Resource Management in NG Wireless Systems, 2001.

SAITO R.; SCHIOZER, R. F.. Uso de Derivativos e Gerenciamento de Risco em Empresas Não Financeiras: Uma Comparação entre Evidências Brasileiras e Internacionais. In: ENANPAD, 28, 2004, Curitiba. Anais... Curitiba/PR, ANPAD, 2004, 1, CD-ROM.

SAITO R.; SCHIOZER, R. F.. Determinantes da Gestão de Risco em Empresas de Capital Aberto no Brasil. In: ENANPAD, 29, 2005, Brasília/DF. Anais... Brasília/DF, ANPAD, 2005, 1, CD-ROM.

SHARPE, W. F.. Capital asset prices: a theory of market equilibrium under conditions of risk. Journal of Finance, 1964.

SILVEIRA, H. P.; BARROS, L.A.; FAMA, R.. Conceito de Taxa Livre de Risco e sua Aplicação no Capital Asset Pricing Model - Um Estudo Exploratório para o Mercado Brasileiro. In: $2^{\circ}$ Encontro Brasileiro de Finanças, 2002, Rio de Janeiro. Anais do $2^{\circ}$ Encontro Brasileiro de Finanças, 2002.

SOUZA, A. B.. Projetos de investimentos-de capital: elaboração, análise, tomada de decisão, São Paulo: Atlas, 2003.

TELÓ, A., R.. Desempenho Organizacional: Planejamento Financeiro em Empresas Familiares. Revista FAE, v.4, n.1, p.17-26, 2001

TRIGEORGIS, L.. Real options: managerial flexibility and strategy in resource allocation, Cambridge, MA: MIT Press, 1996.

YOUNG, S. D.; O.BYRNE, S. F.. EVA e gestão baseada em valor: guia prático para implementação, Porto Alegre: Bookman, 2003.

\section{NOTA - Endereço dos autores}

Universidade Potiguar

Campus Floriano Peixoto

Av. Floriano Peixoto, 295 - Petrópolis

Natal - RN

501012-500

Universidade Federal da Paraíba

Departamento de Estatística - CCEN

Cidade Universitária - João Pessoa - PB

58000-000
Universidade Federal do Rio de Janeiro

COPPE - UFRJ

Ilha do Fundão

Rio de Janeiro - RJ

21941-972 